\title{
Stat proteins and oncogenesis
}

\author{
Jacqueline Bromberg \\ Memorial Sloan-Kettering Cancer Center, 1230 York Avenue, Box 397, New York, New York 10021, USA. \\ Phone: (212) 639-2577; Fax: (646) 422-2045; E-mail: bromberj@mskcc.org.
}

J. Clin. Invest. 109:1139-1142 (2002). DOI:10.1172/JCI200215617.

The discovery of Stat proteins' key role in IFN signaling, initially described over ten years ago, provided the first molecular link of growth factor receptor stimulation to the direct activation of a transcription factor (1). Since that time a large number of growth factor receptors and some nonreceptor tyrosine kinases have been found to lead to the activation of these transcription factors (2).

The contributions of individual Stat proteins to normal cytokine signaling and development have been studied in various cell culture systems and in vivo in mice made deficient for one or more of these proteins (3). This approach has identified some related roles, as well as many unique, nonoverlapping physiological roles, for the various members of the Stat family. In summary, Stat1-deficient mice are unable to respond to IFNs and are subsequently susceptible to bacterial and viral pathogens. Likewise, disruption of Stat 2 gives rise to animals unable to respond to type 1 IFNs, with increased susceptibility to viral infections (see Candotti et al., this Perspective series, ref. 4). Stat4- and Stat6-deficient animals reveal a requirement for IL-12- or IL-4-mediated proliferation of T cells, respectively (see Decker et al., this series, ref. 5). The phenotypes of Stat5A and Stat5B individual knockouts reveal the importance of Stat $5 \mathrm{~A}$ in breast development and lactation and the importance of Stat5B in the development of sexually dimorphic patterns of gene expression within the liver. In addition to these phenotypes, Stat5A/5B double knockouts are abnormal in their T cell and B cell development. Because Stat3-deficient animals die early in embryogenesis, the role of this protein in a number of biological functions had to be determined in conditional knockouts. As discussed by Levy and Lee in this series (6), Stat 3 is implicated in keratinocyte migration, $\mathrm{T}$ cell apoptosis, IL-10-mediated signaling in macrophages, and the induction of apoptosis in the involuting breast.

Beyond these various roles in normal cellular and physiological processes, the Stat proteins are now known to participate in cellular transformation and oncogenesis. Here, I consider the evidence implicating these molecules, particularly Stats 1,3, and 5, in tumor formation and progression.

\section{Stats and tumorigenesis}

During the multistep process of tumorigenesis, cells lose their normal ability to sense and repair DNA dam- age and to regulate cell cycle progression and apoptosis. In parallel, they acquire abnormal patterns of growth factor signaling, angiogenesis, and invasive growth. While the Stats are not known to contribute directly to cell cycle checkpoint regulation or DNA repair, they contribute to tumorigenesis through their intimate connection to growth factor signaling, apoptosis, and angiogenesis. In addition, because these molecules play key roles in immune responses, defective Stat signaling can favor tumor development by compromising immune surveillance.

\section{STAT1 as a tumor suppressor}

STAT1, the first STAT to be discovered, is required for signaling by the IFNs $(7,8)$, which, in addition to their requirement in innate immunity, serve as potent inhibitors of growth and promoters of apoptosis. Although Stat1-deficient mice develop no spontaneous tumors, they are highly susceptible to chemical carcinogen-induced tumorigenesis (9). Crossing the Stat1 mutation into a p53-deficient background yields animals that develop tumors more rapidly, and with a broader spectrum of tumor types, than is seen with $p 53$ single mutants. Because tumors from carcinogen-treated wild-type animals also grow far more rapidly when transplanted into the Stat1-deficient animals than they do in a wild-type host, it appears that Stat 1 is needed for the host's tumor surveillance capabilities (9).

The requirement of Stat 1 for apoptosis and growth arrest (10-14) in some cell types may be explained by its ability to upregulate caspases and the cdk inhibitor p21. Interestingly, p21 upregulation by STAT1 in mammary cells appears to involve BRCA1, which is often lost in familial and other forms of breast cancer (15). Determining whether STAT1 itself is functionally impaired in primary cancers is not an easy task, particularly in solid tumors, where signaling function may be sensitive to the local environment, and ex vivo studies of STAT1 activity in isolated cells are of questionable significance. Nevertheless, it would not be surprising to find that STAT1 is mutated in some human tumors. It will be interesting to determine whether a recently described (16) human STAT1 mutation, found in families with an inherited susceptibility to mycobacterial infections, also leads to an increased risk of cancer. 
Table 1

Constitutive activation of STATs in primary cancers and tumor-derived cell lines

\begin{tabular}{lclc}
\hline Solid tumors & Activated STAT & Liquid tumors & Activated STAT \\
Breast cancer & 1,3 & Chronic myelogenous leukemia & 5 \\
Head and neck cancer & 1,3 & Acute myeloid leukemia & $1,3,5$ \\
Prostate cancer & 3 & Chronic lymphocytic leukemia & 1,3 \\
Melanoma & 3 & Mycosis fungoides & 3 \\
Ovarian cancer & 3 & Acute lymphoblastic leukemia & 1,5 \\
Lung cancer & 1,3 & Erythroleukemia & 1,5 \\
Brain tumors & 1,3 & Burkitt's lymphoma & 3 \\
Pancreatic cancer & 3 & Large granular lymphocyte leukemia & 3 \\
Renal carcinoma & 3 & Myeloma & 3 \\
& & Hodgkins lymphoma & 3 \\
& & Anaplastic large cell lymphoma & 3 \\
Cell lines and/or primary tumor & STAT activation required for transformation & \\
Breast cancer & Required STAT & Oncogenes & Required STAT \\
Head and neck cancer & 3 & src & 3 \\
Prostate cancer & 3 & eyk & 3 \\
Melanoma & 3 & ret & 3 \\
Thyroid cancer & 3 & Ick & 3,5 \\
Myeloma & 3 & Npm-alk & 3 \\
Hodgkins & 3 & & 3 \\
Large granular lymphocyte leukemia & 3 & & \\
Hepatocellular carcinoma & 3 & 3 & \\
\hline
\end{tabular}

Black, Stats constitutively activated in a number of primary liquid and solid tumors; Blue, Stats required for the transformed phenotype of the tumor-derived cell lines, primary tumors, or oncogene transformed cell lines.

\section{Constitutive activation of Stats in cellular transformation}

The first reports of persistently tyrosine-phosphorylated (that is, persistently activated) STAT proteins in primary cancers and tumor-derived cell lines came shortly after the discovery of the STATs (Table 1). Subsequent work showed that, in a number of tumorderived cell lines, the STATs, particularly STAT3, are required to maintain a transformed phenotype. STAT5 is also commonly found to be constitutively activated in certain malignancies, especially leukemias and lymphomas (Table 1). The expression of fusion proteins that cause heightened or unrestrained JAK2, PDGF-R, or ABL signaling can lead to the constitutive activation of STAT5. Work in murine models shows that bcr-abland v-abl-induced leukemias do not require Stat5 (17) but that this protein is required for a myeloproliferative disorder that results from a TEL-JAK fusion (18).

Stat3-deficient murine T cells, mammary epithelial cells, macrophages, fibroblasts, and keratinocytes are viable, relatively normal cells with subtle defects typically involving the regulation of apoptosis (19). Thus, in these few examples, Stat 3 is not essential for viability of normal cells. In contrast, many cancer-derived cell lines that contain consitutively activated STAT3 are dependent on this protein and undergo growth arrest or apoptosis when treated with antisense or dominant negative constructs directed at STAT3.

Direct evidence that STAT3 signaling is oncogenic comes from work with a spontaneously dimerizingmutant form of STAT3, STAT3-C, which does not require tyrosine phosphorylation to be activated yet is capable of transforming fibroblasts (20). There are no known naturally occurring mutations of STAT3 that lead to its constitutive activation and subsequent transformation of cells. In all naturally occurring tumors and in oncogene-transformed cells, STAT3 activation is typically dependent upon dysregulated growth factor receptor tyrosine kinases or their associated Jak kinases. Thus, in the case of thyroid cancers associated with an aberrantly regulated Ret receptor tyrosine kinase, Schuringa et al. (21) have found that transformation by Ret requires phosphorylation and activation of STAT3. Similarly, expression of a dominant negative STAT3 abrogates cellular transformation in the acute myelogenous leukemia (AML) and gastrointestinal stromal cell tumors (GISTs) associated with activating mutations in the receptor c-kit (22). STAT3 is also persistently activated in Hodgkin disease, where AG490, an inhibitor of JAK2 and STAT3 phosphorylation, can be used to inhibit tumor growth (23). In primary prostate cancer specimens and prostate cancer-derived cell lines, likewise, STAT3 is activated, and the introduction of antisense to STAT3 provokes tumor cell apoptosis (24, 25). Finally, persistently activated STAT3 and JAK2 are found in the rare malignancy large granular lymphocyte leukemia; treatments that block STAT3 expression or function cause cancer cell death by upregulating the proapoptotic protein Fas and downregulating the antiapoptotic Mcl-1 $(26,27)$.

Antisense reagents and dominant negative constructs directed at STAT3, as well as Jak inhibitors such as AG490, thus hold the promise of tumor-specific growth inhibition and appear to be useful against a range of cancer cells (Table 1). In addition, Jove and colleagues recently developed a novel approach to blocking STAT3 function, using a phosphopeptide tethered to a protein transduction domain (28). This peptide 
enters cells, recognizes the SH2 domain of STAT3, and prevents de novo STAT3 phosphorylation. Consistent with the requirement of activated STAT3 in cellular transformation, this peptide blocks the transformation of NIH-3T3 cells by v-src (28).

\section{Routes to constitutive STAT activation}

Dysregulated tyrosine kinase activity and increased levels of tyrosine phosphorylation are common in cancer cells and often result from the overexpression of growth factor receptors and their ligands. Salient examples are seen in multiple myeloma, which characteristically overexpresses IL- 6 and its receptor, and in head and neck cancers, where EGF and its receptor are found at high levels (29). Increased expression of a growth factor receptor, such as the PDGF-R in mesotheliomas or gliomas and Erb2 (Her2neu) in breast cancer, is felt to be a critical step in the formation of these cancers. High levels of c-kit may be critical for the abnormal phenotype of GISTs. Excessive Jak kinase activity in such tumors is perhaps the most common mechanism for constitutive phosphorylation and activation of the STATs. The basis of receptor tyrosine kinase or growth factor receptor overexpression is not understood but may involve the loss of internalization and turnover that normally follows ligand binding. Proteins such as the JAKs and c-Src, which associate with these receptors, may also accumulate to abnormal levels and thus may promote STAT hyperactivation in cancer cells.

A number of human malignancies also express the TEL-JAK fusion protein described above, which induces the persistent dimerization and constitutive activation of JAK2 $(18,30-32)$. The JAK2 inhibitor AG490, first used against leukemia cells in which the kinase is constitutively active (33), has more recently been found to induce growth arrest or apoptosis in many tumor-derived cell lines, suggesting that this kinase is important in a variety of cancers (34). Endogenous inhibitors of the JAKs include SHP1 and SHP2, as well as the SOCS proteins, STAT-regulated factors that feed back to suppress the upstream Jaks (35). Interestingly, Yoshikawa et al. recently showed that methylation-induced silencing of the SOCS1 gene leads to constitutive STAT3 activation in a hepatocellular carcinoma-derived cell line (36).

After the dimeric Stat has entered the nucleus and has bound its target DNA, it is eventually dephosphorylated by a still-unidentified tyrosine phosphatase and shuttles back to the cytoplasm. These interactions suggest still other mechanisms by which the normal regulation of Stat signaling might be lost in tumorigenesis, some of which have indeed been demonstrated in cancer cells or

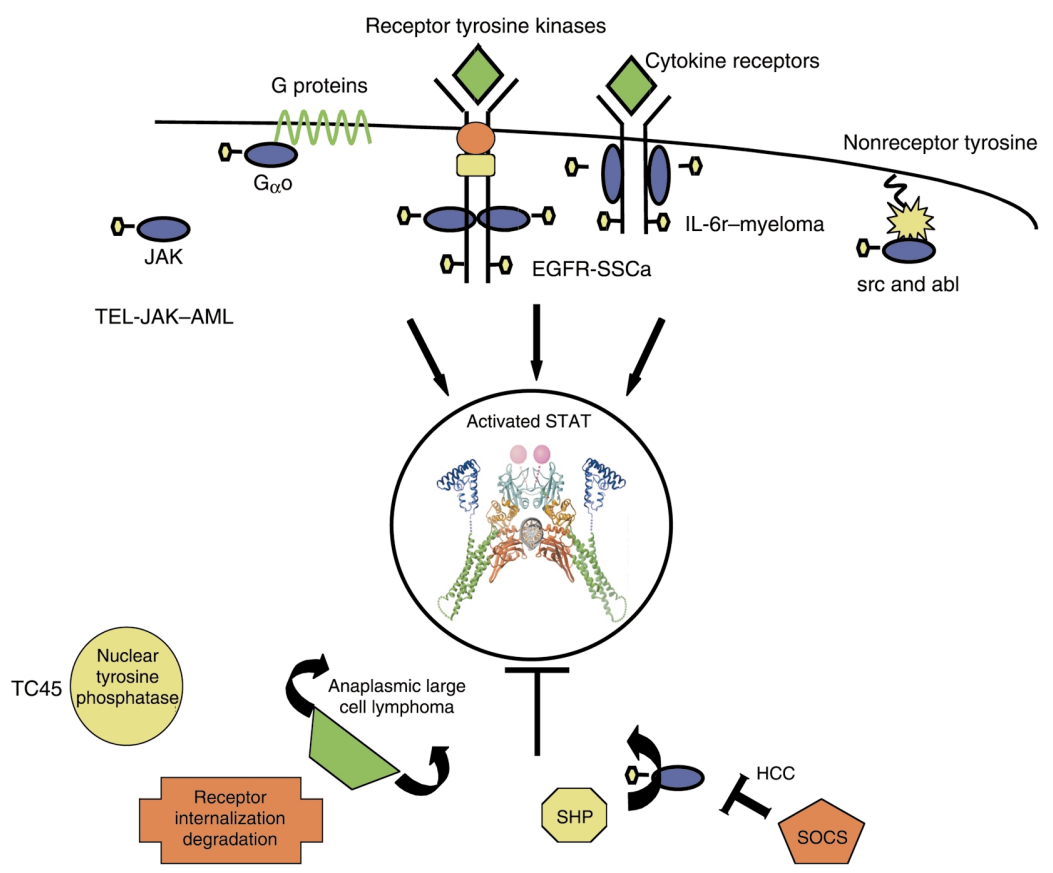

Figure 1

Routes to persistent activation of Stats in cancer cells. Stat proteins, in particular Stat3, are persistently activated in a wide variety of different cancers. Explanations for Stat activation are potentially quite varied and include over-expression or dysregulation of kinases or inhibition of the negative regulators. Some examples are listed in red. Jak kinases are indicated as blue ovals. The TEL-JAK fusion protein is constitutively active as a kinase and leads to AML through its effects on STATs 1, 3, or 5. The oncogenic G-protein subunit $\mathrm{G}_{\alpha} \mathrm{O}$, associates with Jak proteins, leading to Stat 3 hyperactivation and the transformation of NIH3T3. Overexpression and dysregulation of the EGFR and its ligand TGF- $\alpha$ stimulates the receptor's intrinsic tyrosine kinase activity and those of the associate Jak and Src protein kinases, thereby inducing persistent Stat 3 activity in squamous cell carcinomas. Overexpression and dysregulation of the IL- 6 receptor and production of its ligand IL-6 stimulates Stat 3 activity in multiple myeloma. Abnormal regulation of the oncogenic proteins Src and Abl likewise causes excess. Stat3 phosphorylation in sarcomas and chronic myelogenous leukemia, among other malignancies. SOCS1 gene methylation and silencing constitutively activates JAKs1 and 2 and leads to persistent STAT3 phosphorylation in hepatocellular carcinomas. Loss of PIAS3 expression allows for unregulated STAT3 activity in anaplastic large cell lymphoma. 
tumor-derived cell lines (Figure 1). In particular, a group of STAT inhibitors known as PIAS proteins have been identified that bind tyrosine-phosphorylated, dimeric STATs and prevent them from binding DNA, perhaps by potentiating tyrosine dephosphorylation (37). Downregulation of PIAS3 protein, as is seen in anaplastic large cell lymphoma, may be in part responsible for maintaining high levels of activated STAT3 (38).

\section{Concluding thoughts}

Cellular transformation by activated Stat 3 and its relatives undoubtedly occurs through the transcriptional regulation of specific genes. Many Stat 3 target genes are known, including those encoding the antiapoptotic proteins $\mathrm{Bcl}-\mathrm{xl}, \mathrm{Mcl}-1$, and $\mathrm{Bcl}-2$, the proliferation-associated proteins Cyclin D1 and Myc, and the proangiogenic factor VEGF $(20,27,29,39-41)$. In addition, STAT3 cooperates with c-Jun to repress expression of FAS, presumably interfering with cancer cell apoptosis (42). Still other genes must be regulated indirectly by STAT3, many of which may contribute to oncogenesis or tumor progression.

It is important to recognize that Stats, including Stat 3 , can be persistently activated under various circumstances in which cellular transformation is not the ultimate phenotype - in macrophages within an inflamed joint, for example, and in neuronal hypoxia (43-45). Because tumorigenesis is a multistep process, constitutive activation of Stat proteins alone need not lead to transformation. However, in the appropriate context it clearly is a critical molecule in tumorigenesis. The growing list of cases where suppression of STAT signaling leads to the demise of tumor cells establishes that these molecules contribute to the cancerous phenotype and provides hope that the near future will bring therapeutics targeting activated STAT molecules.

1. Darnell, J.E., Jr. 1997. STATs and gene regulation. Science. 277:1630-1635.

2. Bromberg, J., and Darnell, J.E., Jr. 2000. The role of STATs in transcriptional control and their impact on cellular function. Oncogene 19:2468-2473.

3. Levy, D.E. 1999. Physiological significance of STAT proteins: investigations through gene disruption in vivo. Cell. Mol. Life Sci. 55:1559-1567.

4. Candotti, F., Notarangelo, L., Visconti, R., and O'Shea, J. 2002. Molecular aspects of primary immunodeficiencies. J. Clin. Invest. In press.

5. Decker, T., Stockinger, S., Karaghiosoff, M., Müller, M., and Kovarik, P. 2002. Interferons and Stats in innate immunity to microorganisms. J. Clin. Invest. In press.

6. Levy, D.E., and Lee, C. 2002. What does Stat3 do? J. Clin. Invest. 109:1143-1148. DOI:10.1172/JCI200215650.

7. Meraz, M.A., et al. 1996. Targeted disruption of the Stat 1 gene in mice reveals unexpected physiologic specificity in the JAK-STAT signaling pathway. Cell. 84:431-442.

8. Durbin, J.E., Hackenmiller, R., Simon, M.C., and Levy, D.E. 1996. Targeted disruption of the mouse Stat 1 gene results in compromised innate immunity to viral disease. Cell. 84:443-450.

9. Kaplan, D.H., et al. 1998. Demonstration of an interferon gamma-dependent tumor surveillance system in immunocompetent mice. Proc. Natl. Acad. Sci. USA. 95:7556-7561.

10. Bromberg, J.F., Horvath, C.M., Wen, Z., Schreiber, R.D., and Darnell, J.E., Jr. 1996. Transcriptionally active Stat 1 is required for the antiproliferative effects of both IFN- $\alpha$ and IFN- $\gamma$. Proc. Natl. Acad. Sci. USA. 93:7673-7678.

11. Kumar, A., Commane, M., Flickinger, T.W., Horvath, C.M., and Stark, G.R. 1997. Defective TNF-alpha-induced apoptosis in STAT1-null cells due to low constitutive levels of caspases. Science. 278:1630-1632.

12. Lee, C.K., Smith, E., Gimeno, R., Gertner, R., and Levy, D.E. 2000. STAT1 affects lymphocyte survival and proliferation partially independent of its role downstream of IFN-gamma. J. Immunol. 164:1286-1292.

13. Chin, Y.E., et al. 1996. Cell growth arrest and induction of cyclin-depend- ent kinase inhibitor p21 WAF1/CIP1 mediated by STAT1. Science. 272:719-722.

14. Bromberg, J.F., Fan, Z., Brown, C., Mendelsohn, J., and Darnell, J.E., Jr. 1998. Epidermal growth factor-induced growth inhibition requires Stat activation. Cell Growth Differ. 9:505-512.

15. Ouchi, T., Lee, S.W., Ouchi, M., Aaronson, S.A., and Horvath, C.M. 2000 Collaboration of signal transducer and activator of transcription 1 (STAT1) and BRCA1 in differential regulation of IFN-gamma target genes. Proc. Natl. Acad. Sci. USA. 97:5208-5213.

16. Dupuis, S., et al. 2001. Impairment of mycobacterial but not viral immunity by a germline human STAT1 mutation. Science. 293:300-303.

17. Sexl, V., et al. 2000. Stat5a/b contribute to interleukin 7-induced B-cell precursor expansion, but abl- and bcr/abl-induced transformation are independent of stat5. Blood. 96:2277-2283.

18. Schwaller, J., et al. 2000. Stat5 is essential for the myelo- and lymphoproliferative disease induced by TEL/JAK2. Mol. Cell. 6:693-704.

19. Akira, S. 2000. Roles of STAT3 defined by tissue-specific gene targeting. Oncogene. 19:2607-2611.

20. Bromberg, J., et al. 1999. Stat3 as an oncogene. Cell. 98:295-303.

21. Schuringa, J.J., et al. 2001. MEN2A-RET-induced cellular transformation by activation of STAT3. Oncogene. 20:5350-5358.

22. Ning, Z.Q., Li, J., McGuinness, M., and Arceci, R.J. 2001. STAT3 activation is required for $\operatorname{Asp}(816)$ mutant c-Kit induced tumorigenicity. Oncogene. 20:4528-4536.

23. Kube, D., et al. 2001. STAT3 is constitutively activated in Hodgkin cell lines. Blood. 98:762-770.

24. Gao, B., et al. 2001. Constitutive activation of JAK-STAT3 signaling by BRCA1 in human prostate cancer cells. FEBS Lett. 488:179-184.

25. Campbell, C.L., Jiang, Z., Savarese, D.M., and Savarese, T.M. 2001 Increased expression of the interleukin-11 receptor and evidence of STAT3 activation in prostate carcinoma. Am. J. Pathol. 158:25-32.

26. Epling-Burnette, P.K., et al. 2001. Inhibition of STAT3 signaling leads to apoptosis of leukemic large granular lymphocytes and decreased Mcl-1 expression. J. Clin. Invest. 107:351-362.

27. Epling-Burnette, P.K., et al. 2001. Cooperative regulation of Mcl-1 by Janus kinase/stat and phosphatidylinositol 3-kinase contribute to granulocytemacrophage colony-stimulating factor-delayed apoptosis in human neutrophils. J. Immunol. 166:7486-7495.

28. Turkson, J., et al. 2001. Phosphotyrosyl peptides block Stat3-mediated DNA binding activity, gene regulation, and cell transformation. J. Biol. Chem. 276:45443-45455

29. Bowman, T., Garcia, R., Turkson, J., and Jove, R. 2000. STATs in oncogenesis. Oncogene. 19:2474-2488.

30. Lacronique, V., et al. 1997. A TEL-JAK2 fusion protein with constitutive kinase activity in human leukemia. Science. 278:1309-1312.

31. Peeters, P., et al. 1997. Fusion of TEL, the ETS-variant gene 6 (ETV6), to the receptor-associated kinase JAK2 as a result of $\mathrm{t}(9 ; 12)$ in a lymphoid and $\mathrm{t}(9 ; 15 ; 12)$ in a myeloid leukemia. Blood. 90:2535-2540.

32. Lacronique, V., et al. 2000. Transforming properties of chimeric TEL-JAK proteins in Ba/F3 cells. Blood. 95:2076-2083.

33. Meydan, N., et al. 1996. Inhibition of acute lymphoblastic leukaemia by a Jak-2 inhibitor. Nature. 379:645-648.

34. Turkson, J., and Jove, R. 2000. STAT proteins: novel molecular targets for cancer drug discovery. Oncogene. 19:6613-6626.

35. Hilton, D.J. 1999. Negative regulators of cytokine signal transduction. Cell. Mol. Life Sci. 55:1568-1577.

36. Yoshikawa, H., et al. 2001. SOCS-1, a negative regulator of the JAK/STAT pathway, is silenced by methylation in human hepatocellular carcinoma and shows growth-suppression activity. Nat. Genet. 28:29-35.

37. Shuai, K. 2000. Modulation of STAT signaling by STAT-interacting proteins. Oncogene. 19:2638-2644.

38. Zhang, Q., et al. 2002. Multilevel dysregulation of STAT3 activation in anaplastic lymphoma kinase-positive T/null-cell lymphoma. J. Immunol. 168:466-474.

39. Bowman, T., et al. 2001. Stat3-mediated Myc expression is required for Src transformation and PDGF-induced mitogenesis. Proc. Natl. Acad. Sci. USA 98:7319-7324.

40. Catlett-Falcone, R., et al. 1999. Constitutive activation of Stat3 signaling confers resistance to apoptosis in human U266 myeloma cells. Immunity. 10:105-115

41. Niu, G., et al. 2002. Constitutive Stat3 activity up-regulates VEGF expression and tumor angiogenesis. Proc. Natl. Acad. Sci. USA. In press.

42. Ivanov, V.N., et al. 2001. Cooperation between STAT3 and c-jun suppresses Fas transcription. Mol. Cell. 7:517-528.

43. Wen, T.C., Peng, H., Hata, R., Desaki, J., and Sakanaka, M. 2001. Induction of phosphorylated-Stat 3 following focal cerebral ischemia in mice. Neurosci. Lett. 303:153-156.

44. Ivashkiv, L.B. 2000. Jak-STAT signaling pathways in cells of the immune system. Rev. Immunogenet. 2:220-230.

45. Deon, D., et al. 2001. Cross-talk between IL-1 and IL-6 signaling pathways in rheumatoid arthritis synovial fibroblasts. J. Immunol. 167:5395-5403. 